\title{
"Yellow-dragon Wonderful-seed Formula" for hyperuricemia in gout patients with dampness-heat pouring downward pattern: a pilot randomized controlled trial
}

Xiao Ning Yu ${ }^{1+}$, Hai Yan $\mathrm{Wu}^{2+}$, Yuan Ping Deng ${ }^{3+}$, Guang Tong Zhuang ${ }^{4 \dagger}$, Bang Huan Tan ${ }^{5}$, Yan Zhou Huang ${ }^{5}$, Shi Yun Tang ${ }^{6}$, Xiang Tu ${ }^{7^{*}}$, James B Jordan ${ }^{7}$ and Sen Zhong ${ }^{8^{*}}$

\begin{abstract}
Background: In Traditional Chinese Medicine (TCM) theories, the typical clinical manifestations of gout are attributed to the "dampness-heat pouring downward." Therefore, TCM practitioners always consider prescribing the formulae which are believed to clear heat and drain dampness for the management of gout. This clinical trial aims: (1) to determine the hypouricemic effect of "Yellow-dragon Wonderful-seed Formula" (YWF) decoction in gout patients with dampness-heat pouring downward pattern and (2) to determine if gypsum could provide additional significant benefits to YWF.
\end{abstract}

Methods: A total of 72 hyperuricemic individuals with gout and dampness-heat pouring downward pattern were included with 62 of them completing the trial. Participants were randomly assigned to the YWF group, the YWF + gypsum group, or the allopurinol group. YWF and YWF + gypsum decoctions were orally administered for four weeks. Allopurinol was also orally administered for four weeks as the active control. Serum uric acid (sUA) level was the primary outcome measure. Urine urate level, scores on the SF-36 scale, erythrocyte sedimentation rate (ESR), $\mathrm{X}$ ray film, and $\mathrm{C}$ reactive protein (CRP) level were the secondary outcome measures.

Results: Compared with the values at week $0, Y W F$ and YWF + gypsum did not significantly decrease the sUA level at each weekend reading. YWF, YWF + gypsum, and allopurinol decreased the urine urate levels and there were significant differences between the YWF group and the YWF + gypsum group. All the changes in the eight structures of SF-36 during the intervention period were not significantly different among the three groups and there was no significant difference in the CRP level among the three groups at each weekend reading.

Conclusions: YWM, which modified on the basis of Two Wonderful Herbs Powder (2WHP), does not show significant hypouricemic effect. There is a possibility that Gypsum Fibrosum may provide additional effects to YWF in decreasing the urine urate levels but cannot add benefits to YWF in other outcome measures.

Trial registration: Chinese Clinical Trial Registry, ChiCTR-TRC-12001933. Registered on 10 February 2012.

Keywords: Yellow-dragon Wonderful-seed formula, Chinese herbal medicine formula, Gout, Hyperuricemia, Randomized controlled trial, Gypsum Fibrosum

\footnotetext{
*Correspondence: tuxiang2005@163.com; zhongsen6606@163.com

${ }^{+}$Xiao Ning Yu, Hai Yan Wu, Yuan Ping Deng and Guang Tong Zhuang contributed equally to this work.

${ }^{7}$ National Traditional Chinese Medicine Clinical Research Base for Diabetes

Mellitus/Teaching Hospital of Chengdu University of Traditional Chinese

Medicine, Chengdu 610072, Sichuan Province, China

${ }^{8}$ Administration Committee of Affiliated Hospitals of Chengdu University of

Traditional Chinese Medicine, Chengdu 611137, Sichuan Province, China

Full list of author information is available at the end of the article
}

(c) The Author(s). 2018 Open Access This article is distributed under the terms of the Creative Commons Attribution 4.0 International License (http://creativecommons.org/licenses/by/4.0/), which permits unrestricted use, distribution, and reproduction in any medium, provided you give appropriate credit to the original author(s) and the source, provide a link to the Creative Commons license, and indicate if changes were made. The Creative Commons Public Domain Dedication waiver (http://creativecommons.org/publicdomain/zero/1.0/) applies to the data made available in this article, unless otherwise stated. 


\section{Background}

Epidemiological evidence from New Zealand, the USA, the UK, and China suggests that gout is becoming more prevalent [1]. The incidence of gout is approximately $1-2 \%$ in western countries, affecting $>3 \%$ of adults in the US [1, 2], which is very similar to that in China (1\%) [3]. Gout is commonly characterized by hyperuricemia and acute arthritis, tophi, interstitial nephritis, and joint deformity resulting from deposition of sodium urate crystals.

At the beginning of the 21st century, according to the epidemiology of the eastern city of Nanjing (Jiangsu province), the mean concentration of serum uric acid (sUA) was $342.3 \pm 87.3 \mu \mathrm{mol} / \mathrm{L}$ in Chinese men and $252.2 \pm$ $77.8 \mu \mathrm{mol} / \mathrm{L}$ in Chinese women [4]. The incidence of hyperuricemia was $17.6 \%$ in men and $9.3 \%$ in women; gout occurred in $1.98 \%$ and $0.72 \%$, respectively [4].

An investigation carried out in the northern province of Hebei in 2004 reported that the incidence of hyperuricemia was $15.8 \%$ in 1215 male individuals [5]. An article published in 2012 reported that the incidence of hyperuricemia was $24.73 \%$ in 1929 men and $10.79 \%$ in 686 women in Guizhou province, located in south China [6].

In Traditional Chinese Medicine (TCM) theories, the typical clinical manifestations of gout are attributed to the "dampness-heat pouring downward." Both dampness and heat are the pathogenic factors and they have distinct characteristics: dampness is similar to water and heat is similar to fire. When heat invades gout patients, it results in a red, swollen joint. In the original Chinese medical text, the Huangdi Neijing says, "When dampness attacks the body, it first impairs the lower part of the body." Dampness tends to move downward, like water. Therefore, the disease caused by dampness usually involves the lower part of the body. Dampness usually affects the lower extremities in gout patients, leading to attacks on the feet, ankle, or knees.

Therefore, TCM practitioners always consider prescribing the formulae which are believed to clear heat and drain dampness for the management of gout. Theoretically, dampness can be drained by diuretic TCM or dried by using TCM bitter in taste. Heat can be eliminated by TCM of cold property.

The typical diuretic TCM formula is Two Wonderful Herbs Powder (2WHP). In fact, there is a series of formulae developed from the $2 W H P$ - the Three Wonderful Herbs Pill (3WHP) is modified from the $2 W H P$ by adding one TCM (Cyathulae Radix) and the Four Wonderful Herbs Pill ( $4 \mathrm{WHP})$ is also modified from the $2 \mathrm{WHP}$ by adding two TCM (Cyathulae Radix and Coix seeds).

Current reports demonstrated that the series formulae modified from $2 \mathrm{WHP}$ are effective for hyperuricemia in gout (see below).
2WHP:

The experiment in vivo by Kong et al. [7] demonstrated that the hypouricemic effect of $2 W H P$ was "equal to that of the reference drug allopurinol" in hyperuricemic mice pretreated with oxonate.

In the article by Li [8], 98 individuals with gout received modified 2 WHP for 14 days. The results showed that the levels of sUA decreased below normal values in 64 participants, accounting for $65.3 \%$. Lv et al. [9] reported that the water extract of $2 W H P$ could inhibit the production of hepatic urate and promote the excretion of urine urate in hyperuricemic rats induced by oxonic acid potassium salt. The underlying mechanisms may be related to the downregulation of the messenger RNA (mRNA) and protein levels of hepatic xanthine oxidase (XOD) and renal mouse urate transporter 1 (mURAT1) regulated by $2 W H P$.

3WHP:

When expanding $2 W H P$ to $3 W H P$ by adding Cyathulae Radix, some case reports showed positive results. Lu et al. [10] reported that modified $3 W H P$ was given to 30 gout patients for ten days and the sUA values of 25 patients decreased below normal limits and were maintained for at least two years. Shan et al. [11] carried out an in vivo study to explore the mechanisms of 3 WHP for inflammation of acute gouty arthritis. Their results showed that $3 W H P$ could reduce the inflammation in the ankle of rats with acute gouty arthritis and this was related to the inhibition of TNF- $\alpha$, IL- 6 , and IL- 8 levels in the ankle synovial tissues of gout rats which were associated with the downregulation of NF-KB P65 protein expression by 3 WHP.

4WHP:

$\mathrm{Hu}$ et al. [12] reported 4 WHP could enhance the renal urate excretion by effectively reversing oxonate-induced alterations in renal mURAT1, mouse glucose transporter 9 (mGLUT9), and organic anion transporter 1 (mOAT1) mRNA and protein levels. The conclusion was that their findings "suggest that $4 \mathrm{WHP}$ processes uricosuric and nephroprotective actions by regulating renal organic ion transporters in hyperuricemic animals."

In a randomized controlled trial (RCT), 178 participants were randomized to either the modified $4 W H P$ group or the western medicine group. The treatment period was 14 days. The sUA level of the 4 WHP group decreased from $585.93 \pm 93.93 \mu \mathrm{mol} / \mathrm{L}$ to $319.13 \pm$ $87.63 \mu \mathrm{mol} / \mathrm{L}$. There was no significant difference between the $4 W H P$ group and western medicine group. Two individuals suffered from slight diarrhea in the 4WHP group [13]. In another RCT, 120 participants were randomly assigned to either the modified $4 \mathrm{WHP}$ group $(n=60)$ or to the allopurinol group $(n=60)$. The UA and CRP levels were determined after a one-month intervention period. The authors concluded that modified 
4WHP could "significantly improve the symptoms and signs of gouty arthritis and decrease the levels of UA and CRP. It is good for gouty arthritis" [14].

As mentioned above, modified 2 WHP appears to be an excellent approach to hyperuricemia in gout patients. Published literature also suggested that gypsum (Pinyin Name: Shi Gao; Latin Name: Gypsum Fibrosum) played an important role for hyperuricemia in gout patients [15-17]. Generally, current clinical evidence concerning TCM for gout is methodologically problematic $[8,10$, $13,14]$. We decided to put the 2 WHP and other TCM together to create a new formula, i.e. $Y W F$, and test its clinical efficacy with a RCT of desirable methodology.

\section{Methods}

\section{Aims}

We carried out a RCT to evaluate YWF for the treatment of hyperuricemia in gout patients with the specific TCM pattern, i.e. dampness-heat pouring downward pattern. This was also to determine whether Gypsum Fibrosum could provide significant additional benefits to YWF.

\section{Design and setting of the study}

The present clinical study was a pilot RCT where there were three arms and the allocation ratio among the three groups was 1:1:1. This pilot trial was neither a superiority test nor a non-inferiority test, it was an exploratory trial. The pilot RCT was conducted at six centers in Sichuan Province and Chongqing City, China.

\section{Participants}

Participants must have a physician diagnosis of gout [18] and hyperuricemia (sUA $>420 \mu \mathrm{mol} / \mathrm{L}$ ) and be aged 18 70 years. If the patient had already received treatment for gout, he/she had to undergo a two-week washout period; only those whose sUA remained $>420 \mu \mathrm{mol} / \mathrm{L}$ after the washout period could be included. Only the patients differentiated as dampness-heat pouring downward pattern were included. The dampness-heat pouring downward pattern was confirmed by clinical symptoms and signs manifested as red, swollen, hot, and painful acute joint arthritis, red tongue, yellow and greasy tongue coating, and smooth pulse [19]. The pattern must be differentiated by at least two trained doctors of TCM. Patients were excluded if they had any of the following: pregnancy or lactation; allergic constitution, or an allergic history to test TCM or allopurinol; serum creatinine $>1.5 \mathrm{mg} / \mathrm{dL}$; elevated values of ALT twice as high as the normal upper limit; severe deformity or stiffness of gouty arthropathy resulting in disability; arrhythmia of clinical significance; or a history of alcohol abuse. The patients were also excluded if they had severe cerebrovascular, kidney, liver, or hematopoietic system co-morbidities, cancer or mental disorders; taken concurrent hypouricemic medications, azathioprine, 6-mercaptopurine, medications containing aspirin $(>325 \mathrm{mg}$ ) or salicylate; or had participated in other clinical trials within the past three months.

\section{Interventions}

The typical TCM formula to clear heat and dry dampness is Three Kinds of Kernels Decoction (TKKD) and cardamom is the monarch herb of TKKD. We therefore, added cardamom to $4 W H P$ to make the new formula become more appropriate for the dampness-heat pouring downward pattern. Because TCM experts believe that blood stasis plays an important role in gout, we added Pheretima to promote blood circulation and to dredge the collateral. The TCM ingredient of the new formula is shown in Table 1 and we call it "Yellow-dragon Wonderful-seed Formula" (YWF), a reference to Phellodendron bark in Chinese which is called "Yellow cypress" and Pheretima called "earth dragon." As some TCM experts reported, gypsum plays a special role in the treatment of gout [16, 17]. We designed an independent arm with YWF + gypsum. The daily dose of gypsum was $15 \mathrm{~g}$.

In this study, 72 individuals were randomly assigned to receive either YWF decoction, YWF + gypsum decoction, or allopurinol. All patients were advised not to consume purine-rich diets. TCM was purchased from Sichuan Rejuvenation Hall Pharmaceutical Co., Ltd., China. Professor HouLin Xia from the College Pharmacy, Chengdu University of TCM, confirmed that all TCM ingredients were the right herbal species recorded in the Chinese Pharmacopeia (2015 Edition) [20]. Suining (Sichuan, China) FDA assayed the quality standards of all ingredients TCM and provided quality reports which confirmed that their quality standards meet the Chinese Pharmacopeia (2015 Edition) requirements. All TCM ingredients were decocted together for $30 \mathrm{~min}$ at $120^{\circ} \mathrm{C}$ with an automatic boiling and packaging machine, using three packages of decoction (100 mL/package). The TCM decoction was taken orally three times daily (100 mL each time) for four weeks. The starting dose of allopurinol was $100 \mathrm{mg} \mathrm{day}^{-1}$ in week 1 and increased to $200 \mathrm{mg}$ during weeks $2-4$.

\section{Outcomes}

The level of sUA was the primary outcome, which was measured by enzymatic method with a Beckman (USA, AU5400) automatic biochemical analyzer every four weeks. The assay agent was purchased from ZhongSheng Corporation, Beijing, China. The secondary outcome measures included the urine urate measured every four weeks by the same analyzer and agent for sUA, scores on SF-36 which were measured at week 0 and week 4, the ESR was measured by the Westergren method every four weeks, 
Table 1 Ingredients TCM of YWF

\begin{tabular}{llll}
\hline Pinyin name & Latin name & English name & Daily dose (g) \\
\hline Di Long & Pheretima & Earthworm & 10 \\
Dou Kou & Amomum kravanh Pirre ex Gagnep. & Amomum, cardamon & 6 \\
Huang Bai & Cortex Phellodendri Chinensis & Cortex Phellodendri, Phellodendron bark & 10 \\
Cang Zhu & Atractylodes Lancea (Thunb.) DC. & Atractylodes, sword-like attractylodes & rhizome, Chineseatractylodes rhizome \\
Yi Yi Ren & Coix lacryma-jobi L.var.ma-yuen & Coix seeds,Job's tears & \\
Chuan Niu Xi & (Roman.)Stapf(Yi Yi) & Cyathula, medicinal cyathula root & 20
\end{tabular}

the CRP was measured with a CRP automatic analyzer (QUIKREAD GO, Finland) and its matched agent kits every four weeks, and the $\mathrm{X}$ ray film examined at week 0 and week 4. We recorded acute flares during the treatment period. We designed serum concentration of xanthine and hypoxanthine as the additional outcome measures in the study protocol, but they were not assayed due to the insufficiency of funding.

\section{Sample size}

As this study was a pilot trial, each arm of the RCT had 24 participants.

\section{Randomization}

\section{Sequence allocation}

The randomization sequence was generated with an SAS software.

\section{Allocation concealment}

The randomization sequence was concealed and disseminated with opaque envelopes.

\section{Implementation}

The Good Clinical Practice (GCP) center of the Teaching Hospital of Chengdu University of TCM provided the randomization sequence. TCM doctors at six collaborating centers enrolled patients and the assistant nurses disseminated the randomization envelopes.

\section{Blinding}

Blinding was not used due to the obvious difference between YWF decoction and allopurinol. However, the statisticians were blind to the study design.

\section{Statistical methods}

Intention-to-treat (ITT) analysis was used to analyze data and the primary population for assessing efficacy was the full-analysis set (FAS). Patients who took at least one dose of the study medications and had at least one value on treatment were included by the FAS. The last-observation-carried-forward method was used to impute the missing data, whereby missing values were replaced by the last non-missing value. The baseline characteristics among the three groups were analyzed by one-way analysis of variance (ANOVA). Repeated measures and multivariate analysis of variance of the general linear model were used to determine the changes in sUA, urine urate, and CRP. The scores on SF-36 scale were analyzed by analysis of covariance. SPSS software (version 18.0, SPSS Inc., Chicago, IL, USA) was used to analyze statistics and $P<0.05$ was considered significant.

\section{Results}

\section{Participant flow}

A total of 72 male participants were involved in this study and ten dropped out during the study period (Fig. 1).

\section{Recruitment}

The recruitment period was from 1 March 2012 to 15 December 2014.

\section{Baseline data}

The baseline characteristics of the three groups of patients were comparable $(P>0.05)$ and shown in Table 2.

\section{Numbers analyzed}

The per-protocol set (PPS) included 21 participants in the YWF arm, 20 in the YWF + gypsum arm, and 21 in the allopurinol arm. The FAS included 24 participants in each of the three arms.

\section{Outcomes}

The data analysis on the FAS was largely consistent with the PPS.

\section{sUA}

Compared with the values at week 0 , YWF and YWF + gypsum did not significantly decrease the sUA levels at each weekend reading (Table 3, Fig. 2). There was no significant difference between YWF and YWF + gypsum at each reading $(P>0.05)$. 


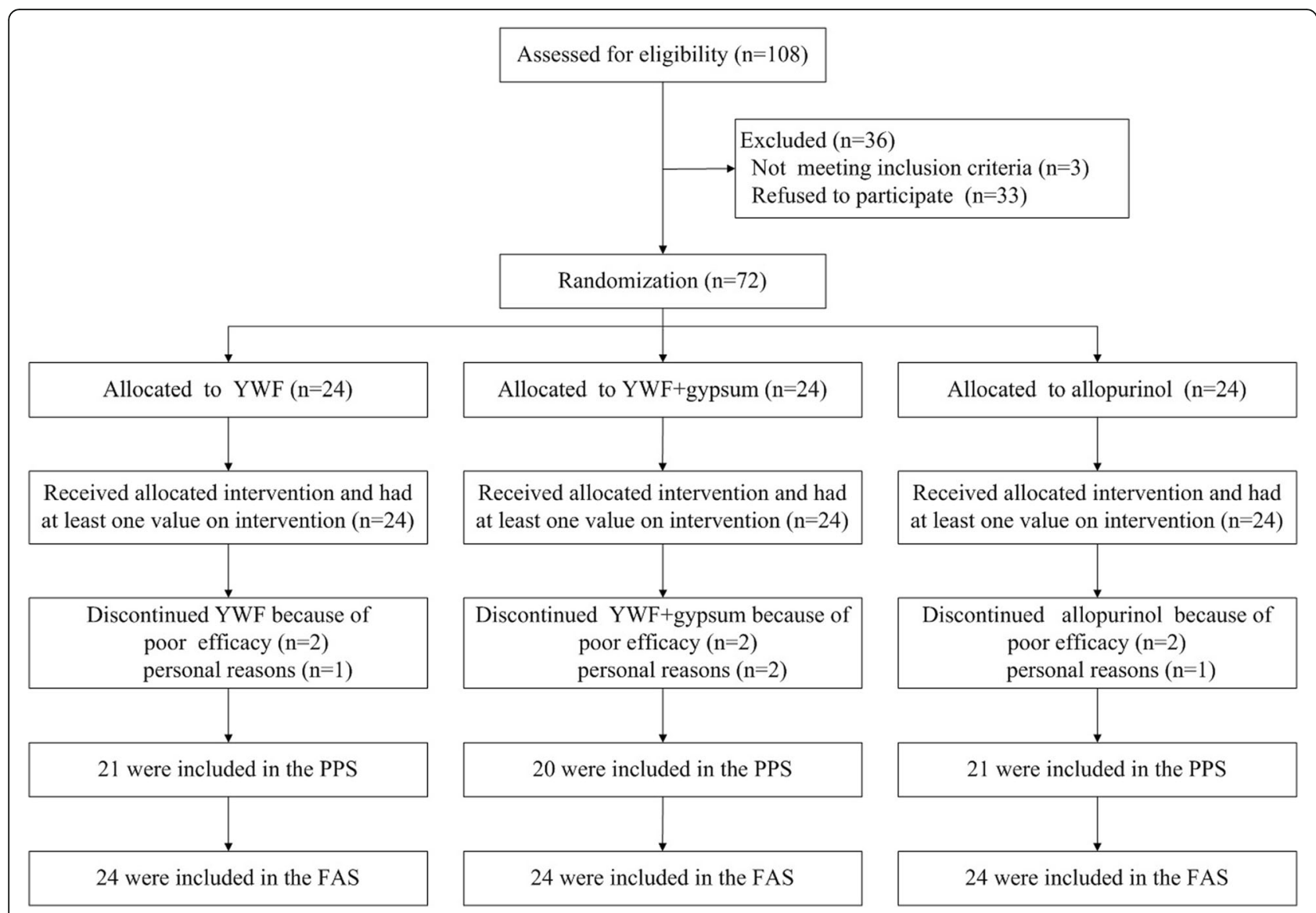

Fig. 1 Participant flow. A total of 72 patients were randomized to the three arms. All 72 participants received the allocated intervention and therefore all 72 were included in the FAS. Ten individuals dropped out and therefore 62 participants were included in the PPS

Table 2 The baseline characteristics of the FAS population of the three groups of patients

\begin{tabular}{|c|c|c|c|c|c|}
\hline \multicolumn{2}{|l|}{ Characteristic } & YWF & YWF + gypsum & Allopurinol & $F$ value \\
\hline \multicolumn{2}{|l|}{ Age (years) } & $45.33 \pm 9.86$ & $46.13 \pm 10.75$ & $49.21 \pm 9.47$ & 1.00 \\
\hline \multicolumn{2}{|c|}{ Gout history (months) } & $39.42 \pm 29.00$ & $55.25 \pm 36.58$ & $42.96 \pm 32.38$ & 1.54 \\
\hline \multicolumn{2}{|l|}{$s \cup A(\mu m o l / L)$} & $562.29 \pm 108.29$ & $585.46 \pm 100.06$ & $618.00 \pm 114.27$ & 1.62 \\
\hline \multicolumn{2}{|c|}{ Urine urate $(\mathrm{mmol} / 24 \mathrm{~h})$} & $7.80 \pm 0.37$ & $7.79 \pm 0.33$ & $7.78 \pm 0.31$ & 0.01 \\
\hline \multicolumn{2}{|l|}{$\mathrm{CRP}(\mathrm{mg} / \mathrm{L})$} & $13.13 \pm 2.63$ & $14.03 \pm 3.40$ & $13.15 \pm 1.13$ & 0.33 \\
\hline \multicolumn{2}{|l|}{$\operatorname{ESR}(\mathrm{mm} / \mathrm{h})$} & $8.96 \pm 4.99$ & $8.79 \pm 3.16$ & $9.88 \pm 6.96$ & 0.29 \\
\hline \multirow[t]{8}{*}{ SF-36 scale } & $\mathrm{BP}$ & $25.00 \pm 14.52$ & $31.71 \pm 11.74$ & $26.96 \pm 9.54$ & 1.95 \\
\hline & $\mathrm{RP}$ & $27.08 \pm 23.22$ & $22.92 \pm 19.39$ & $25.00 \pm 23.31$ & 0.21 \\
\hline & PF & $78.96 \pm 7.07$ & $81.04 \pm 9.44$ & $77.50 \pm 10.11$ & 0.95 \\
\hline & VT & $73.13 \pm 7.91$ & $75.83 \pm 9.85$ & $75.42 \pm 9.20$ & 0.63 \\
\hline & SF & $41.15 \pm 22.87$ & $43.23 \pm 17.28$ & $42.71 \pm 20.16$ & 0.07 \\
\hline & RE & $19.43 \pm 21.79$ & $26.38 \pm 24.04$ & $24.99 \pm 22.52$ & 0.95 \\
\hline & $\mathrm{GH}$ & $64.79 \pm 13.95$ & $66.04 \pm 12.70$ & $59.71 \pm 14.43$ & 1.44 \\
\hline & $\mathrm{MH}$ & $43.67 \pm 15.78$ & $46.17 \pm 15.78$ & $46.42 \pm 16.23$ & 0.22 \\
\hline
\end{tabular}


Table 3 Changes in SUA (mean $\pm \mathrm{SD}, \mu \mathrm{mol} / \mathrm{L}$ )

\begin{tabular}{|c|c|c|c|c|c|c|}
\hline & & Week 0 & Week 1 & Week 2 & Week 3 & Week 4 \\
\hline \multirow[t]{3}{*}{ PPS } & YWF arm & $547.38 \pm 97.32$ & $539.24 \pm 121.62$ & $522.43 \pm 143.97$ & $516.29 \pm 155.89$ & $517.38 \pm 157.96$ \\
\hline & YWF + gypsum arm & $570.35 \pm 93.61$ & $572.90 \pm 148.29$ & $568.05 \pm 140.46$ & $548.75 \pm 164.78$ & $559.10 \pm 202.94$ \\
\hline & Allopurinol arm & $618.76 \pm 115.64$ & $517.43 \pm 124.03^{*}$ & $482.10 \pm 144.27^{*}$ & $476.24 \pm 136.72^{*}$ & $466.81 \pm 141.27^{*}$ \\
\hline \multirow[t]{3}{*}{ FAS } & YWF arm & $562.29 \pm 108.30$ & $550.33 \pm 120.16$ & $530.71 \pm 143.63$ & $525.33 \pm 154.39$ & $526.29 \pm 156.15$ \\
\hline & YWF + gypsum arm & $585.46 \pm 100.06$ & $588.62 \pm 144.79$ & $574.50 \pm 157.62$ & $557.67 \pm 176.13$ & $566.29 \pm 206.08$ \\
\hline & Allopurinol arm & $618.00 \pm 114.27$ & $528.46 \pm 125.58^{*}$ & $494.21 \pm 145.64^{*}$ & $489.08 \pm 139.65^{*}$ & $480.83 \pm 144.34^{*}$ \\
\hline
\end{tabular}

*In comparison with week 0 , there was a statistically significant difference $(P<0.05)$

\section{Urine urate}

The levels of urine urate decreased in all three groups $(P<0.05)$. Analysis on FAS showed that there was a significant difference between the YWF group and the YWF + gypsum group at each reading $(P<0.05$, Table 4 , Fig. 3).

\section{SF-36}

The items of the SF-36 scale can be summarized as eight structures including physical functioning (PF), role-physical $(\mathrm{RP})$, bodily pain $(\mathrm{BP})$, general health $(\mathrm{GH})$, vitality $(\mathrm{VT})$, social functioning (SF), role-emotional (RE), and mental health $(\mathrm{MH})$. All the changes in the eight structures of SF-36 during the treatment period were not significantly different between the three groups (data not shown).

\section{CRP}

In comparison with the values at week 0 , the CRP level of the YWF significantly decreased at week 4 ( $P$ $<0.05)$ and there was no significant difference among the three groups at each weekend reading (Table 5).

\section{ESR}

The ESR levels of the three arms fell in the scope of normal values and there was no significant change during treatment period (data not shown).

\section{$X$-ray film}

No individuals showed any significant changes on X-ray film digital image.

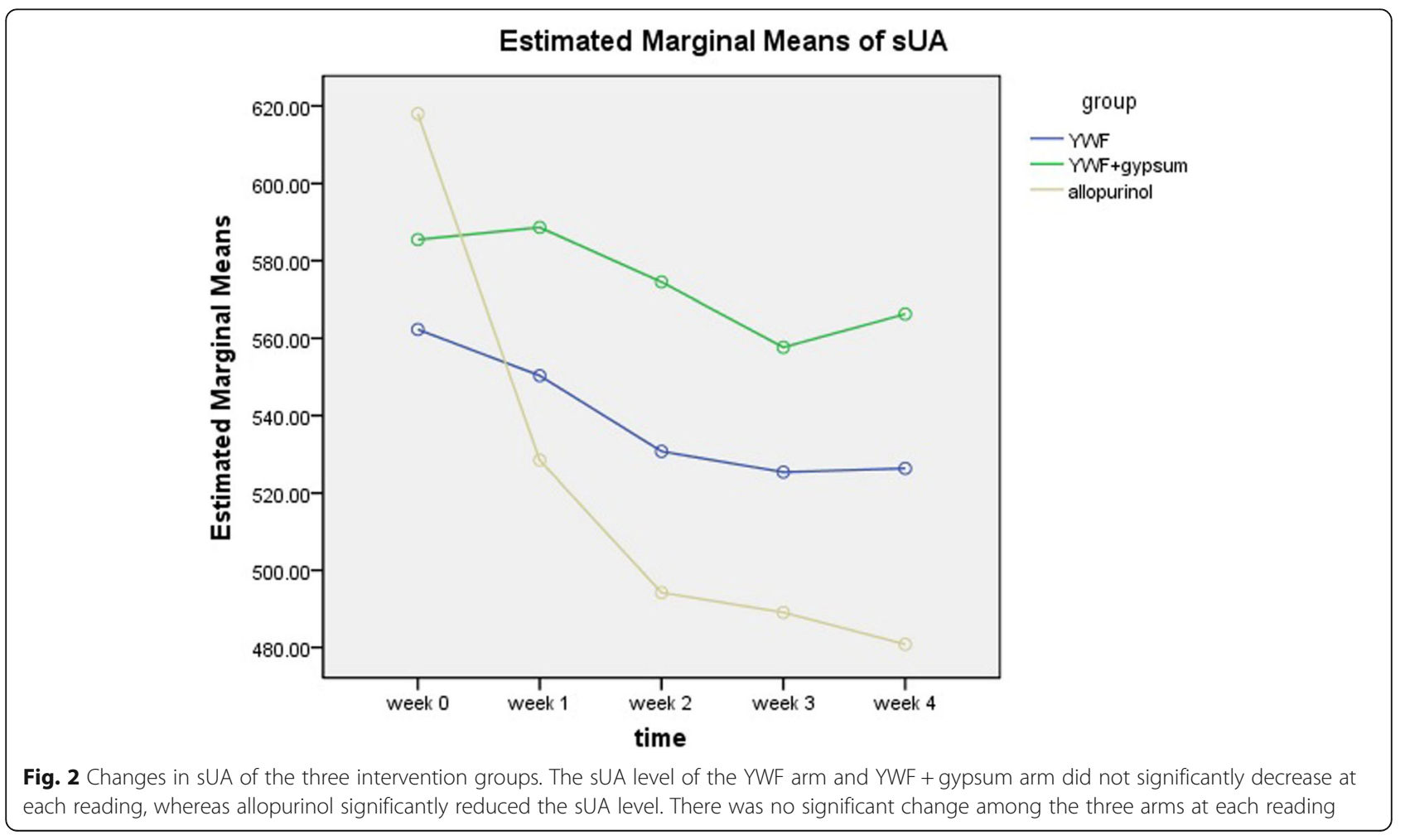


Table 4 Changes in urine urate (mean \pm SD, mmol/24 h)

\begin{tabular}{lllllll}
\hline & & Week 0 & Week 1 & Week 2 & Week 3 & Week 4 \\
\hline PPS & YWF arm & $7.77 \pm 0.39$ & $7.59 \pm 0.28^{*}$ & $7.42 \pm 0.32^{*}$ & $7.33 \pm 0.32^{*}$ & $7.33 \pm 0.35^{*}$ \\
& YWF + gypsum arm & $7.82 \pm 0.28$ & $7.50 \pm 0.33^{*}$ & $7.28 \pm 0.44^{*}$ & $7.19 \pm 0.45^{*}$ & $7.17 \pm 0.48^{*}$ \\
& Allopurinol arm & $7.80 \pm 0.31$ & $7.53 \pm 0.27^{*}$ & $7.32 \pm 0.36^{*}$ & $7.24 \pm 0.39^{*}$ & $7.21 \pm 0.41^{*}$ \\
FAS & YWF arm & $7.80 \pm 0.37$ & $7.65 \pm 0.31^{*}$ & $7.50 \pm 0.36^{*}$ & $7.43 \pm 0.38^{*}$ & $7.43 \pm 0.41^{*}$ \\
& YWF + gypsum arm & $7.79 \pm 0.33$ & $7.48 \pm 0.30^{*+}$ & $7.28 \pm 0.40^{*+}$ & $7.21 \pm 0.41^{*^{+}}$ & $7.19 \pm 0.44^{*+}$ \\
& Allopurinol arm & $7.78 \pm 0.31$ & $7.53 \pm 0.26^{*}$ & $7.33 \pm 0.35^{*}$ & $7.25 \pm 0.36^{*}$ & $7.23 \pm 0.40^{*}$ \\
\hline
\end{tabular}

*In comparison with value at week 0 , there was statistically significant difference $(P<0.05)$

${ }^{\dagger}$ In comparison with YWF arm at each reading, there was statistically significant difference $(P<0.05)$

\section{Acute flares}

No acute flare was recorded in each group during treatment period.

\section{Adverse events}

No adverse events were reported.

\section{Discussion}

\section{Limitations}

The lack of blinding of the present clinical study could result in performance bias and detection bias. Another limitation is that we were not sure whether our negative results resulted from the YWF had not been modified as an appropriate formula for patients with long gout history.
We notice that the gout history in our study is, on average, 39-55 months. We consider whether the dampness and heat might have evolved as a "toxin." The toxin accumulated in the blood and joints, resulting in hyperuricemia and gout arthritis. Modifying YWF by adding some TCM with property of detoxification may produce better clinical benefits of YWF.

\section{Generalizability}

Our results of data analysis on FAS were largely consistent with those on PPS, which suggests that our results were sound. To our knowledge, in the field of TCM for gout, it is the first time that a clinical study was presented according to the CONSORT Extension

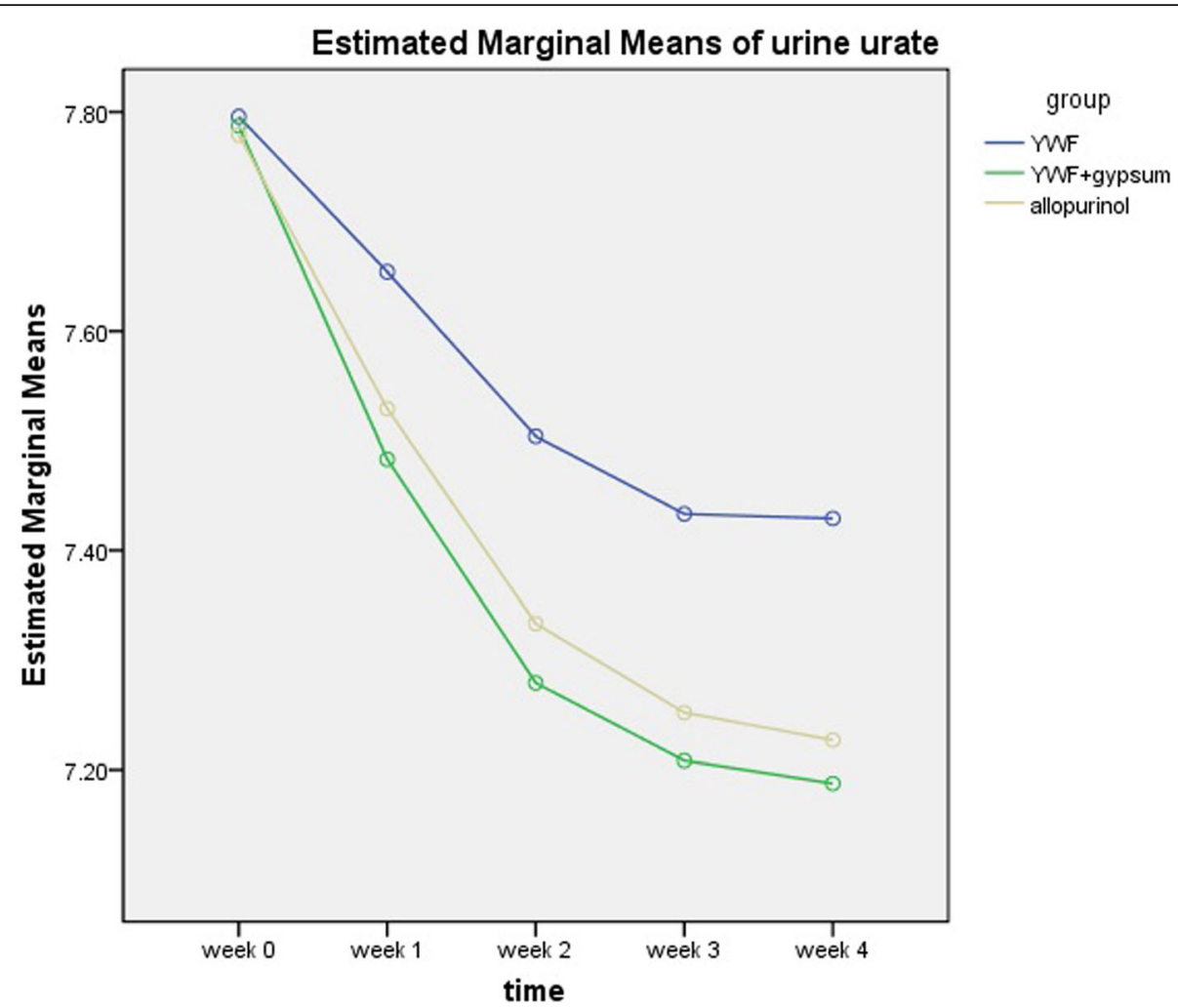

Fig. 3 Changes in urine urate of the three intervention groups. The level of urine urate of all three arms significantly decreased at each reading in comparison with the values at week 0 . There was a significant difference between YWF arm and YWF + gypsum arm at each reading 
Table 5 Changes in CRP (mean $\pm \mathrm{SD}$, mg/L)

\begin{tabular}{lllllll}
\hline & Week 0 & Week 1 & Week 2 & Week 3 & Week 4 \\
\hline PPS & YWF arm & $14.43 \pm 2.73$ & $13.13 \pm 1.82$ & $13.34 \pm 1.08$ & $12.49 \pm 0.64$ & $10.09 \pm 4.71^{*}$ \\
& YWF + gypsum arm & $13.66 \pm 1.63$ & $13.26 \pm 1.81$ & $12.98 \pm 0.75$ & $12.55 \pm 0.85$ & $11.56 \pm 5.06$ \\
& Allopurinol arm & $13.25 \pm 1.32$ & $12.99 \pm 1.19$ & $13.34 \pm 1.15$ & $12.56 \pm 0.77^{*}$ & $11.03 \pm 4.98$ \\
FAS & YWF arm & $13.13 \pm 2.63$ & $12.87 \pm 1.83$ & $13.04 \pm 1.30$ & $12.33 \pm 0.85$ & $10.33 \pm 4.34^{*}$ \\
& YWF + gypsum arm & $14.03 \pm 3.40$ & $13.03 \pm 1.81$ & $12.81 \pm 0.96$ & $12.45 \pm 0.97$ & $11.64 \pm 4.62$ \\
& Allopurinol arm & $13.15 \pm 1.13$ & $13.05 \pm 1.31$ & $13.20 \pm 1.19$ & $12.54 \pm 0.76^{*}$ & $11.13 \pm 4.77$ \\
\hline
\end{tabular}

*In comparison with value at week 0 , there was a statistically significant difference $(P<0.05)$

for Chinese Herbal Medicine Formulas 2017 ([21], Additional file 1). Although the modification of $2 W H P$ may vary greatly due to the large number of TCM, this study is of clinical importance to those many TCM professionals who want to prescribe or receive the formulae modified, based on $2 W H P$.

\section{Interpretation}

A large amount of literature has investigated the conventional urate lowering medications for hyperuricemia in gout, such as febuxostat, allopurinol, and benzbromarone [22]. Febuxostat, given its established efficacy and safety, has been recommended as a suitable pharmacological option for first-line treatment of gout [23]. However, controversies remain: a systematic review and meta-analysis concluded that "There was no evidence that febuxostat is superior to allopurinol for clinically relevant outcomes. Given its higher cost, febuxostat should not be routinely used for chronic gout" [24]. Chen et al. [25] reported a traditional Chinese medicine prescription: Quzhuotongbi decoction could significantly lower the sUA levels in hyperuricemia model rats. Kodithuwakku et al. [26] reported that Shuang Qi gout capsules might be a potent anti-hyperuricemic agent. A systematic review concluded that Chinese herbal decoction and traditional western medicine led to similar clinical efficacy including lowering sUA [27].

A number of animal experiments reported that TCM showed hypouricemic effects $[28,29]$ and 2 WHP series formulae $[14,16,17]$ lowered the uric acid levels by diverse mechanisms. In a systematic review published in 2013, " 25 out of 41 trials, involving 23 different herbal prescriptions, $\mathrm{Li}$, et al., found statistical significance in lowering the serum uric acid level." However, the authors admitted that the evidence was not decent due to "low quality of included trials" [30]. Generally speaking, in humans, the current literature reports TCM shows at least similar, if not better, clinical efficacy in comparison with western medicine [27]. We have carried out a systematic review where 19 RCTs and 1646 participants were included to evaluate the clinical efficacy of $2 W H P$ series formulae and the results show that, compared with western medicine, the TCM formulae based on the
2 WHP series could significantly decrease the level of sUA and increase the cure rate [31]. However, unexpectedly, the results of the present pilot RCT were not consistent with our previous systematic review findings: YWF failed to significantly decrease the sUA levels.

A large number of published clinical studies did not have the control arm [13, 15]. Many published RCTs claimed randomization, but none of them provide the details with respect to randomization. Due to the poor methodology involved in proper randomization, it appears that the current clinical evidence is not sound.

\section{Conclusions}

The present clinical study, as a pilot trial, was exploratory rather than conclusive. We drew the following conclusions based on our research results: (1) YWM when modified on the basis of 2 WHP does not show significant hypouricemic effect; (2) Gypsum Fibrosum could provide additional effects to YWF in decreasing the urine urate levels but cannot add benefits to YWF in other outcome measures. Generally speaking, our clinical study is of optimal methodology and our conclusions were of desirable reliability.

\section{Additional file}

Additional file 1: CONSORT Herbal checklist. (DOCX $15 \mathrm{~kb}$ )

\section{Abbreviations}

2WHP: Two Wonderful Herbs Powder; 3WHP: Three Wonderful Herbs Pill; 4WHP: Four Wonderful Herbs Pill; ANOVA: Analysis of variance; BP: Bodily pain; CRP: C-reactive protein; ESR: Erythrocyte sedimentation rate; FAS: Full-analysis set; GCP: Good Clinical Practice; GH: General health; ITT: Intention-to-treat; mGLUT9: Mouse glucose transporter 9; MH: Mental health; mOAT1: Mouse organic anion transporter 1; mURAT1: Mouse urate transporter 1; PF: Physical functioning; PPS: Per-protocol set; RCT: Randomized controlled trial; RE: Roleemotional; RP: Role-physical; SF: Social functioning; sUA: Serum uric acid; TCM: Traditional Chinese Medicine; TKKD: Three Kinds of Kernels Decoction; VT: Vitality; XOD: Xanthine oxidase; YWF: Yellow-dragon Wonderful-seed Formula

\section{Acknowledgements}

We thank all the people who have been involved in our study.

\section{Funding}

This clinical study is supported by Physician Investigator Foundation of National TCM Clinical Research Base for Diabetes Mellitus (CSZYJ2011010). 
The funding body played no role in the design of the study and collection, analysis, and interpretation of data and in writing the manuscript.

\section{Availability of data and materials}

The datasets used and/or analyzed during the current study are available from the corresponding author on reasonable request.

\section{Authors' contributions}

YXN, WHY, DYP, ZGT, JJB, TX, and ZS conceived this clinical study. TX and ZS named the formula. WHY, DYP, ZGT, TBH and TSY carried out this clinical study. HYZ and TX analyzed the data. YXN, WHY, DYP, ZGT, JJB, TX, and ZS drafted the manuscript. All authors have read and approved the final manuscript.

\section{Ethics approval and consent to participate}

The protocol was approved by the ethics committee of the Teaching Hospital of Chengdu University of TCM and all patients provided a written informed consent.

\section{Consent for publication}

Not applicable.

\section{Competing interests}

The authors declare that they have no competing interests.

\section{Publisher's Note}

Springer Nature remains neutral with regard to jurisdictional claims in published maps and institutional affiliations.

\section{Author details}

${ }^{1}$ Basic Medical College of Chengdu University of Traditional Chinese Medicine, Chengdu 611137, Sichuan Province, China. ${ }^{2}$ Department of Gerontology, Sichuan Academy of Medical Sciences \& Sichuan Provincial People's Hospital, Chengdu 610072, Sichuan Province, China. ${ }^{3}$ Department of Internal Medicine, Traditional Chinese Medicine Hospital of Fushun County, Fushun 643200, Sichuan Province, China. ${ }^{4}$ Department of Internal Medicine, Traditional Chinese Medicine Hospital of Pidu District, Chengdu 611730, Sichuan Province, China. ${ }^{5}$ Department of Internal Medicine, Traditional Chinese Medicine Hospital of Kaizhou District, Chongqing City 405400, China. ${ }^{6}$ College of Clinical Medicine, Chengdu University of Traditional Chinese Medicine, Chengdu 610075, Sichuan Province, China. ${ }^{7}$ National Traditional Chinese Medicine Clinical Research Base for Diabetes Mellitus/Teaching Hospital of Chengdu University of Traditional Chinese Medicine, Chengdu 610072, Sichuan Province, China. ${ }^{8}$ Administration Committee of Affiliated Hospitals of Chengdu University of Traditional Chinese Medicine, Chengdu 611137, Sichuan Province, China.

Received: 4 March 2018 Accepted: 12 September 2018

\section{Published online: 11 October 2018}

\section{References}

1. Roddy E, Doherty M. Epidemiology of gout. Arthritis Res Ther. 2010;12(6): 223

2. Roddy E, Choi HK. Epidemiology of gout. Rheum Dis Clin N Am. 2014;40(2): $155-75$

3. Liu R, Han C, Wu D, Xia X, Gu J, Guan H, et al. Prevalence of hyperuricemia and gout in mainland China from 2000 to 2014: a systematic review and meta-analysis. Biomed Res Int. 2015;2015:762820.

4. Shao JH, Mo BQ, Yu RB, Zhong L, Hua L, Xu YC. Epidemiological study on hyperuricemia and gout in community of Nanjing. Chin J Dis Control Prev. 2003;7(4):305-8.

5. Sun LY, Niu XB, Li JB, Yu QJ. Analysis of blood uric acid and lipid and their relation to gout in 1215 males in Cangzhou in 2004. Chinese J Med Lab Technol. 2005;6(2):135-7.

6. Lu JJ, Qian L, Min W, et al. Study on the prevalence of hyperuricemia and metabolic syndrome in the senior of Guizhou. Guizhou Med J. 2012;36(2): 115-8.

7. Kong LD, Yang C, Ge F, Wang HD, Guo YS. A Chinese herbal medicine Ermiao wan reduces serum uric acid level and inhibits liver xanthine dehydrogenase and xanthine oxidase in mice. J Ethnopharmacol. 2004; 93(2-3):325-30.
8. Li CM. Efficacy observation on 98 subjects with gouty arthritis treated with modified Ermiao Powder. Yunnan J Tradit Chinese Med Mater Med. 2012; 33(4):47.

9. LV YZ, Hu QH, Xing W, Zhen OY, Kong LD. Effects of Ermiao Pill water extracts on imbalance of urate levels and its related genes and protein levels in hyperuricemic mice. Chinese Tradit Herb Drugs. 2010;41(3):418-23.

10. Lu JZ, Wu M. Modified Sanmiao Pill in the treatment of 30 patients with gout. Jiangsu J Tradit Chinese Med. 1998;19(11):31.

11. Wei S, Jue HF. On action mechanism of Sanmiao Pill and Medicinal Cyathula Root for inflammatory reaction of acute gouty arthritis. World Chinese Med. 2013;8(2):189-93.

12. Hu QU, Jiao RQ, Wang X, Lv $Y Z$, Kong LD. Simiao pill ameliorates urate underexcretion and renal dysfunction in hyperuricemic mice. J Ethnopharmacol. 2010;128(3):685-92.

13. Wang HJ, Zhang GH, Jun Z, Fan XZ. A clinical study on modified Simiao Powder for acute gouty arthritis and hyperuricemia. J Emerg Tradit Chinese Med. 2009:18(10):1609-10 1626

14. Qiu R, Shen R, Lin D, Chen Y, Ye H. Treatment of 60 cases of gouty arthritis with modified Simiao Tang. J Tradit Chin Med. 2008;28(2):94-7.

15. Zhao F, Guochun L, Yang Y, Shi L, Xu L, Yin L. A network pharmacology approach to determine active ingredients and rationality of herb combinations of modified-Simiaowan for treatment of gout. $J$ Ethnopharmacol. 2015;168:1-16.

16. Wu JX. Gypsum and Sanmiao decoction for the treatment of acute gouty arthritis in 31 subjects. Jiangsu J Tradit Chinese Med. 2014:5:403.

17. Yuan Q. Heavy dose of gypsum for the treatment of acute gouty arthritis in 26 subjects. Yunnan J Tradit Chinese Med Mater Med. 2002;23(2):15-6.

18. Schlesinger N. Diagnosis of Gout: Clinical, Laboratory, and Radiologic Findings. Am J Manag Care. 2005;11:\$443-50.

19. State Administration of Traditional Chinese Medicine of the People's Republic of China. Criteria of Diagnosis and Therapeutic Effect of Internal Diseases and Syndromes in Traditional Chinese Medicine. ZY/T001.1-94.

20. Chinese National Committee of Pharmacopeia. Pharmacopeia of People's Republic of China (2015 Edition). Beijing: China Medical Science Press; 2015

21. Cheng CW, Wu TX, Shang HC, Li YP, Altman DG, Moher D, et al. CONSORT Extension for Chinese Herbal Medicine Formulas 2017: Recommendations, Explanation, and Elaboration. Ann Intern Med. 2017; https://doi.org/10.7326/ M16-2977.

22. Borghi C, Perez-Ruiz F. Urate lowering therapies in the treatment of gout: a systematic review and meta-analysis. Eur Rev Med Pharmacol Sci. 2016;20(5): 983-92.

23. Frampton JE. Febuxostat: a review of its use in the treatment of hyperuricaemia in patients with gout. Drugs. 2015;75(4):427-38 https://doi. org/10.1007/s40265-015-0360-7.

24. Faruque LI, Ehteshami-Afshar A, Wiebe N, Tjosvold L, Homik J, Tonelli M. A systematic review and meta-analysis on the safety and efficacy of febuxostat versus allopurinol in chronic gout. Semin Arthritis Rheum 2013:43(3):367-375. https://doi.org/https://doi.org/10.1016/j.semarthrit. 2013.05 .004$.

25. Chen J, Zhou J, Wei S, Xie Z, Wen C, Xu G. Effect of a traditional Chinese medicine prescription Quzhuotongbi decoction on hyperuricemia model rats studied by using serum metabolomics based on gas chromatographymass spectrometry. J Chromatogr B Analyt Technol Biomed Life Sci. 2016; 1026:272-8 https://doi.org/10.1016/j.jchromb.2015.10.031.

26. Kodithuwakku ND, Feng YD, Zhang YY, Pan M, Fang WR, Li YM. The molecular insight into the antihyperuricemic and renoprotective effect of Shuang Qi gout capsule in mice. J Ethnopharmacol. 2015;163:278-89 https://doi.org/10.1016/j.jep.2015.01.013.

27. Zhou L, Liu L, Liu X, Chen P, Liu L, Zhang Y, et al. Systematic review and meta-analysis of the clinical efficacy and adverse effects of Chinese herbal decoction for the treatment of gout. PLoS One. 2014;9(1):e85008 https://doi. org/10.1371/journal.pone.0085008.

28. Sun WF, Zhu MM, Li J, Zhang XX, Liu YW, Wu XR, et al. Effects of Xie-ZhuoChu-Bi-Fang on miR-34a and URAT1 and their relationship in hyperuricemic mice. J Ethnopharmacol. 2015:161:163-9.

29. Chen J, Zhou J, Wei S, Xie Z, Wen C, Xu G. Effect of traditional Chinese medicine prescription Quzhuotongbi decoction on hyperuricemia model rats studied by using serum metabolomics based on gas chromatographymass spectrometry. J Chromatogr B Analyt Technol Biomed Life Sci. 2016; 1026:272-8. 
30. Li XX, Han M, Wang YY, Liu JP. Chinese herbal medicine for gout: a systematic review of randomized clinical trials. Clin Rheumatol. 2013;32(7): 943-59.

31. Tang SY, Wan XM, Qin J, Bo P, Xiang T, Sen Z. Effectiveness of Ermiao San series of prescriptions for gout: a systematic review. Pharmacol Clin Chinese Mater Med. 2014;30(6):198-202.

Ready to submit your research? Choose BMC and benefit from:

- fast, convenient online submission

- thorough peer review by experienced researchers in your field

- rapid publication on acceptance

- support for research data, including large and complex data types

- gold Open Access which fosters wider collaboration and increased citations

- maximum visibility for your research: over $100 \mathrm{M}$ website views per year

At $\mathrm{BMC}$, research is always in progress.

Learn more biomedcentral.com/submissions 